\title{
Bone destruction, upward migration, and medialisation of rheumatoid shoulder: a 15 year follow up study
}

J T Lehtinen, E A Belt, M J Kauppi, K Kaarela, P P Kuusela, H J Kautiainen, M U K Lehto

Harvard Shoulder Gervice, Massachusett J T Lehtinen

Rheumatism Foundation Hospital, E A Belt

M J Kauppi

P P Kuusela

H J Kautiainen

Tampere University Hospital, Tampere Finland M U K Lehto

Correspondence to: Dr E A Belt, Rheumatis Foundation Hospit Orthopaedics, FIN-18120 Heinola, Finland

eero.beH@reuma.fimnet.fi

Accepted 29 August 2000
Abstract

Objective-To evaluate bone destruction, upward migration, and medialisation of the glenohumeral (GH) joint in a cohort of 74 patients with seropositive and erosive rheumatoid arthritis followed up prospectively.

Methods-At the 15 year follow up 148 shoulders were radiographed by a standard method. Bone destruction in the GH joint was examined from the radiographs by four methods, of which three measured the migration and one the remodelling of the humeral head. The distances from the greater tuberosity of the humeral head to the coracoid process (medialisation distance (MD)) and to the articular surface of the humeral head (GA) have been previously developed to evaluate the preoperative offsets of the arthritic GH joint. Medial displacement index (MI) and upward migration index (UI) have been recently developed to evaluate the destructive pattern of the rheumatoid GH joint. Destruction of the GH joints was assessed by the Larsen method on a scale of 0 to 5 . The relation between the measurements and the grade of destruction of the GH joints was examined. UI was compared with our previous measurements of the subacromial space.

Results-Both the MI and the UI had a negative correlation with the GH joint destruction (Larsen grade), $r=-0.49(95 \%$ CI -0.36 to -0.60$)$ and $r=-0.58(95 \%$ CI -0.46 to -0.68$)$. The UI correlated significantly with the subacromial space, $r=0.90$ ( $95 \%$ CI 0.86 to 0.93$)$. The mean MI and UI measurements of the non-affected joints were within the reported normal variation. The mean MD collapsed between Larsen grades $4(83.0 \mathrm{~mm})$ and $5(65.5$ $\mathrm{mm}$ ). The morphology of the humeral head began to flatten and erode from the grade 3 onwards and medial head destruction was detected at grade 5 .

Conclusions-Medialisation seems to be preceded by upward migration of the humeral head, indicating rotator cuff damage. Symptomatic Larsen grade 3 shoulders should be intensively followed up by clinical and radiological means. If a total shoulder arthroplasty is considered, at at a sufficiently early stage (Larsen 3 and
4), when soft tissue structures responsible for function are still in proper condition and timing of the operative procedure can be well planned.

(Ann Rheum Dis 2001;60:322-326)

Most (67-91\%) patients with rheumatoid arthritis (RA) have shoulder pain, and more than one in five present moderate or severe glenohumeral $(\mathrm{GH})$ joint destruction during the first 15 years from the disease onset. ${ }^{1-3}$

Continuous plain radiographs of the rheumatoid shoulder are the primary diagnostic means of evaluating the glenohumeral joint and planning shoulder arthroplasty. ${ }^{4-6}$ Medial migration and remodelling of the humeral head with medialisation of the $\mathrm{GH}$ joint due to bone are common radiographic findings in rheumatid arthritis (RA) ${ }^{4-13}$ As far as we know, no previous studies have thoroughly measured the relation between GH join destruction and upward migration and medialisation of the humeral head in patients with RA.

This study aimed at analysing the upward migration and medialisation of the humeral head and their relation to rheumatoid destruction of the GH joint in an inception cohort study of 74 patients with RA followed up for 15 years.

\section{Materials and methods}

During the period $1973-75$ a total of 121 patients with recent ( $\leqslant 6$ months) RA were studied at the Rheumatism Foundation Hospial, Heinola, Finland. The selection criteria, data collection strategy, and details of the patients have been described elsewhere. ${ }^{14}{ }^{15} \mathrm{At}$ the three year follow up 102 patients had rheumatoid factor positive, erosive RA. Subsequently, 24 patients died and four patients failed to attend the 15 year follow up. Thus 74 patients ( $18 \mathrm{men}, 56$ women) were the subjects of this study; their age at onset ranged from 17 to 66 , mean 42 (SD 12) years.

Radiographs of all the 148 shoulders were taken at the 15 year follow up as part of a radiographic survey study. The following standard positioning was used: patient supine, turned $20^{\circ}$ to the imaged side, and the arm in external rotation, palm facing upwards. The same trained radiographer confirmed correct positioning and took $x$ ray pictures by the standard technique: the straight (central beam $90^{\circ}$ towards the $\mathrm{GH}$ joint) anteroposterio projection (distance $0.95 \mathrm{~m}$ ) was used with exposure factors of $48 \mathrm{kV}$ and $-63 \mathrm{~mA}$. The 

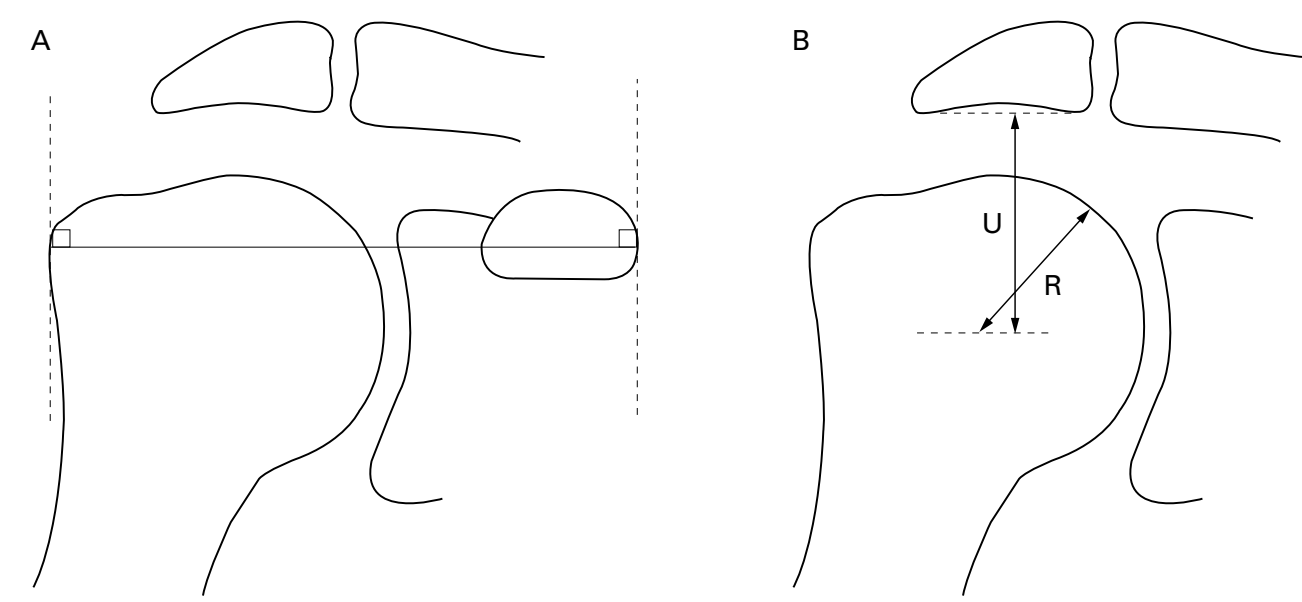

C
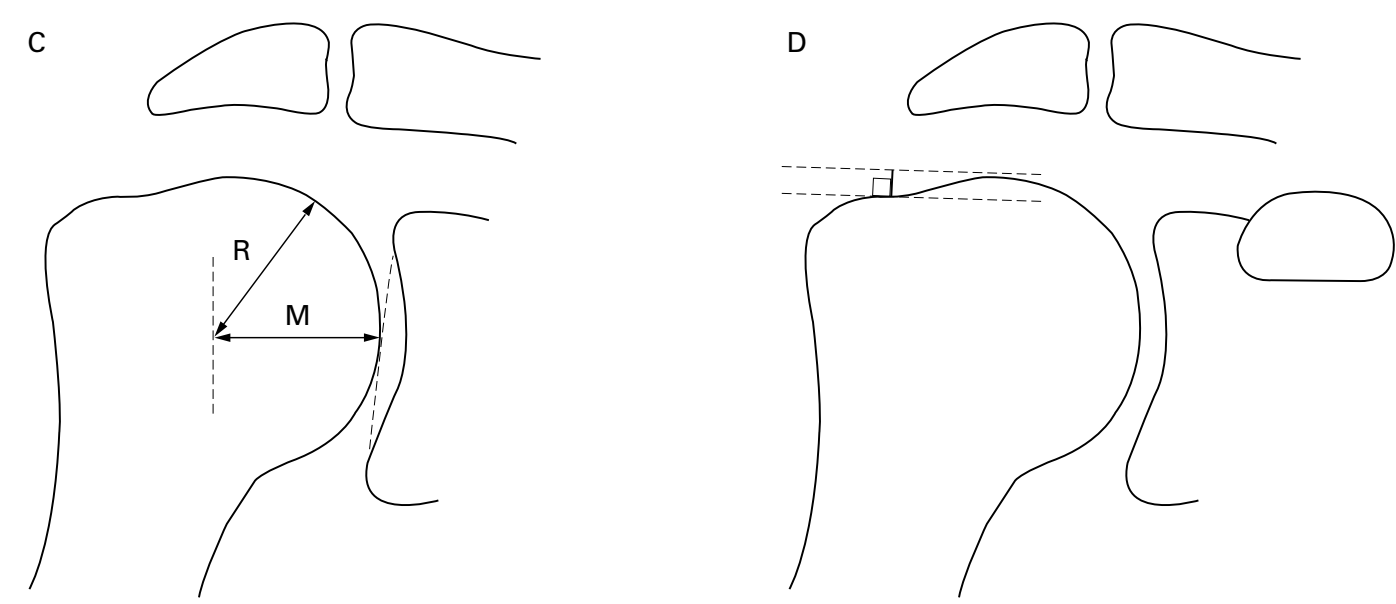

Figure 1 (A-D) Illustration of the measurement methods used in this study. (A) Medialisation distance (MD); (B) upward migration index (UI); (C) medial displacement index $($ ind
$(G A)$. See "Materials and methods" section for further details.

size of the film (Kodak Lanex Regular) was $18 \times 24 \mathrm{~cm}$.

Destruction of the glenohumeral joints was assessed by the Larsen method on a scale of 0 to $5 .^{16}$ One shoulder arthroplasty was performed 13 years after onset of the disease, and in this case the preoperative radiograph in this case the preoperative radiograph (Larsen grade 5) was assessed and measured.
Medialisation (MD) of the GH joint was measured as the distance from the lateral aspect of the greater tuberosity to the medial margin of the coracoid process as described by Figgie et al (fig 1A). ${ }^{17}$ The upward migration index (UI) was obtained by dividing the distance between the centre of the humeral head and the central point of the subacromial head and the central point of the subacromial
surface $(U)$ by the radius of the humeral head surface (U) by the radius of the humeral head
(R) (fig 1B). ${ }^{18}$ The medial displacement index (MI) was obtained by dividing the distance between the centre of the humeral head and the glenoid surface $(\mathrm{M})$ by the radius of the humeral head (R) (fig 1C), as described by Hirooka t $_{\text {al. }}{ }^{18}$ The position of the centre of the humeral head was determined with a circle fitting technique. In shoulders with destruction the bony morphology, the radius of the humeral head, and the original glenoidal and subacromial surfaces were confirmed and measured from the earlier radiographs. Subacromial space measurements from our previous study were compared with the UI. ${ }^{19}$ The perpendicular distance from the superior aspect of the greater tuberosity to the superior articular surface (GA) was measured (fig 1D), as illustrated by Figgie et al. ${ }^{17}$

Statistical comparison between shoulders with different grades of destruction was performed using analysis of variance (ANOVA). Correlations were estimated by Pearson's and Spearman's coefficients. The normality of continuous variables was evaluated by the Kolmogorov-Smirnov statistics with a Lilliefors significance or Shapiro-Wilk statistics. No adjustment was made for multiple testing.

\section{Results}

There was no systematic right-left difference in any of the measuring methods in either sex, and therefore the measurements of the two sides were pooled. Table 1 shows the distribution of the $\mathrm{GH}$ joints $(n=148)$ according to the tor Larsen grading and the mean measurements of estruction in each group, as well as ANOVA tatistics models.

The diminution of the MI between different grades of destruction showed a significant trend $(\mathrm{p}<0.001)$. The correlation coefficient between MI and Larsen grading was -0.49 95\% CI -0.36 to -0.60$)$. The MI correlated with the (6) 0.40 to $0.64)$, though the medialisation occurred late 
Table 1 Radiographic assessment of 148 glenohumeral (GH) joints according to the Larsen grading and the mean medialisation distance $(M D)$, medial displacement index (MI), upward migration index (UI), and greater

\begin{tabular}{|c|c|c|c|c|c|}
\hline Larsen grade & Number of GH joints (\%) & $\begin{array}{l}M D, m m \\
\text { (mean, } S D)\end{array}$ & $M I$ (mean, $S D)$ & $U I$ (mean, $S D)$ & $\begin{array}{l}\text { GA, mm } \\
\text { (mean, } S D,\end{array}$ \\
\hline & $33(22)$ & $85.4(6.3)$ & $0.90(0.04)$ & $1.27(0.06)$ & $6.8(1.7)$ \\
\hline 1 & $44(30)$ & $81.2(6.6)$ & $0.88(0.04)$ & $1.24(0.09)$ & $6.3(1.7)$ \\
\hline 2 & $40(27)$ & $87.9(8.2)$ & $0.87(0.05)$ & $1.20(0.08)$ & $6.8(1.5)$ \\
\hline 3 & $14(9)$ & $85.6(8.1)$ & $0.84(0.08)$ & $1.16(0.14)$ & $6.1(2.3)$ \\
\hline 4 & $6(4)$ & $83.0(4.9)$ & $0.71(0.07)$ & $0.97(0.14)$ & $4.7(1.6)$ \\
\hline 5 & $11(7)$ & $64.5(8.5)$ & $0.49(0.15)$ & $0.82(0.16)$ & $2.9(3.3)$ \\
\hline Total & $148(100)$ & $83.2(9.3)$ & $0.84(0.12)$ & $1.19(0.15)$ & $6.2(2.1)$ \\
\hline \multirow[t]{4}{*}{ ANOVA* } & p Values between: & & & & \\
\hline & & $<0.001$ & $<0.001$ & $<0.001$ & $<0.001$ \\
\hline & $\begin{array}{l}\text { Linearity of destruction } \\
\end{array}$ & $<0.001$ & $<0.001$ & $<0.001$ & $<0.001$ \\
\hline & Eta coefficient & 0.64 & 0.87 & 0.78 & \\
\hline
\end{tabular}

${ }^{\star}$ ANOVA $=$ analysis of variance.

$(\mathrm{p}<0.001)$ and had no significant correlation with Larsen grading. The mean MI of the nonaffected joints (Larsen grades 0 and 1) was 0.89 (SD 0.04). The mean $\mathrm{MD}$ of the non-affected joints was 83.0 (SD 6.7) $\mathrm{mm}$.

The diminution of the UI between increasing grades of destruction had a significant trend $(p<0.001)$. The correlation coefficient between UI and Larsen grading was -0.58 $(95 \% \mathrm{CI}-0.46$ to -0.68$)$. A remarkable correlation occurred between the subacromial space and UI, $r=0.90$ (95\% CI 0.86 to 0.93 ). The mean UI of the non-affected (Larsen grade 0 and 1) joints was 1.26 (SD 0.08).

The GA also decreased with increasing destruction $(\mathrm{p}<0.001)$. There was a slight correlation between GA and Larsen grading, $r=-0.29(95 \% \mathrm{CI}-0.14$ to -0.43$)$. However, it had no significant correlation with either upward migration index or subacromial space measurements.

Discussion

The results of this study confirm the visually observed medialisation in radiographs of rheumatoid shoulder ${ }^{4} 7$ 10-12 The mean distance from the lateral aspect of the humeral head to from the lateral aspect of the humeral head to
the coracoid process collapsed between Larsen the coracoid process collapsed between Larsen
grades 4 and 5 (figs 2 and 3). The medial displacement index (MI) takes into account the size of the bones of the patient by dividing the medial distance by the radius of the humeral head (fig 1C). ${ }^{18}$ It shows more precisely that medialisation is evident already between Larsen grades 3 and 4, before the remarkable reduction between grades 4 and 5 occurs. The mean MI of the non-affected GH joints was within 2SD of the mean of normal controls, as measured by Hirooka et al. ${ }^{18}$ An MD $<70 \mathrm{~mm}$ (mean of non-affected joints - 2SD) could be considered as a sign of definite medialisation of the $\mathrm{GH}$ joint when evaluating a shoulder radiograph taken at $0.95 \mathrm{~mm}$ distance.

The UI correlated well with the subacromial space measurements, emphasising our previous results for the rotator cuff involvement in the rheumatoid shoulder. ${ }^{19}$ The mean UI of the non-affected GH joints was within 2SD of the mean of normal controls, as measured by Hirooka et al. ${ }^{18}$ The previous subacromial space measurements of the non-affected joints were also normal. ${ }^{19}{ }^{20}$ Although upward migration of the humeral head seems to be an ineviable consequence of rheumatoid affection, here seems to be an evident step in rotator cuff there seems to be 作 列 seems to be slightly preceded by superior migration of the humeral head according to ou results (fig 2) and as reported previously. ${ }^{72} 10$ However, if the concavity of the superior glenoidal articular surface is destroyed by eroions, the resultant force from the delto heoretically intact rotator cuff can pull the humeral head into superior subluxation. ${ }^{2}$ Computed tomography measurements have shown that the medial displacement of the glenoid surface was greatest at the upper and middle levels: the surface was inclined superiorly rather than inferiorly. ${ }^{22}$ Yet, at the time of

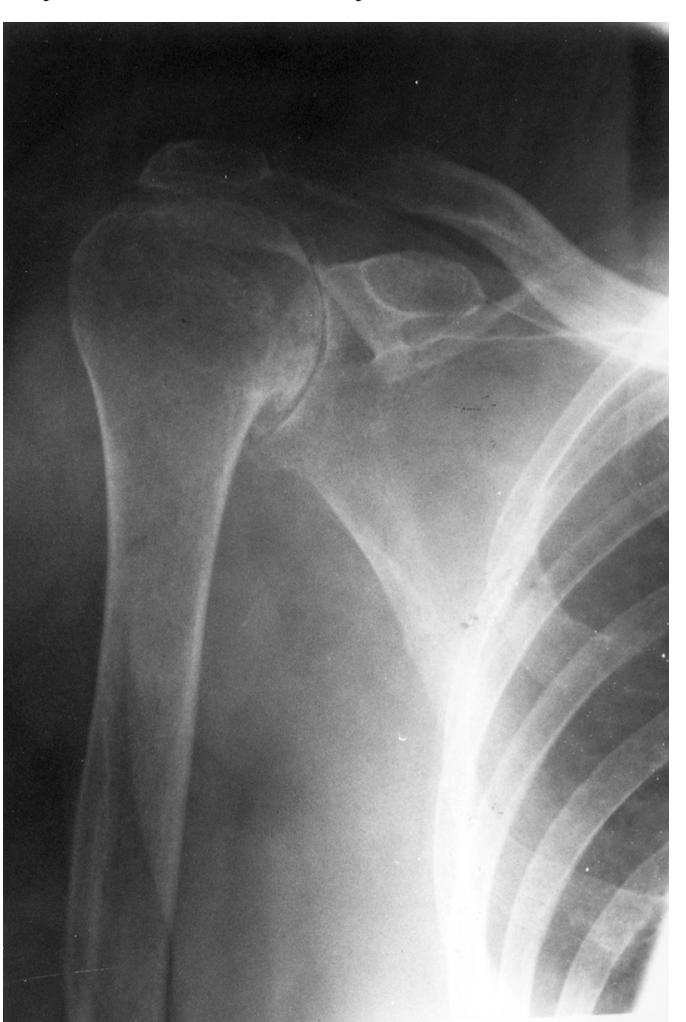
Figure 2 Radiograph of a rheumatoid shoulder. Extensive
erosive destruction on the articular margins, leaving part of the original articular surface still intact (Larsen 4). Eviden superior migration of the humeral head is present and the Jint space has disappeard. However, there is no evident 


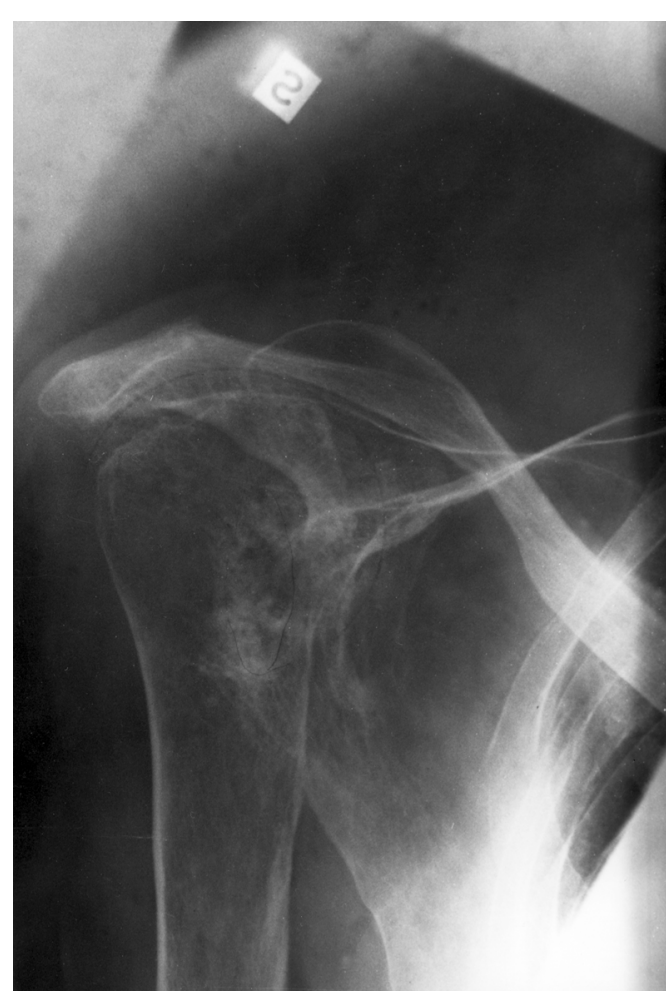

Figure 3 Radiograph of a rheumatoid shoulder. Gross 5). Superior and medial migration of the humeral head have occurred and remarkable loss of the glenoid bone sto is present.

arthroplasty, attenuation or tearing of the rotator cuff is more often found than glenoid destruction, when superior migration of the humeral head is present. ${ }^{102324}$

The commonly described flattening of the humeral head in rheumatoid shoulder is supported by the diminution of the distance between the greater tuberosity and the superior articular surface (GA). ${ }^{8925}$ Flattening is evident in Larsen grade 3 and more obvious in grades 4 and 5, when the superior humeral head is almost even (figs 2 and 3). However, flattening had no correlation with decreasing subacromial space and UI, suggesting that it is not caused by friction with the inferior not caused by friction with the inferior
acromion. This finding is probably caused by erosive destruction, which is more common on the superior joint margin of the humerus. ${ }^{27}$

Shoulder arthroplasty yields excellent pain relief for the painful rheumatoid shoulder. ${ }^{24} 26$ However, the improvement in range of motion and function lag far behind that routinely
expected after hip and knee replacements. ${ }^{23}$ expected after hip and knee replacements.
Restoration of normal glenoid and humeral anatomy in the arthroplasty is correlated with diminished radiographic loosening as well as improved functional results. ${ }^{17}$ Larsen grades 4 and 5, and sometimes grade 3, have been idely and sometion widely, shoulder arthroplasty. An interesting finding is the collapse of the medialisation distance when $\mathrm{GH}$ destruction reaches the Larsen grade of 5 (fig 3). A similar phenomenon occurred with the subacromial space between grades 3 and 4 (fig 2). ${ }^{19}$ Rapid upward migration has been considered as a sign of rapid destruction of the rheumatoid shoulder, therefore early surgical intervention is recommended for these patients. ${ }^{72} 28$ Our results support these findings; upward migration is followed by medialisation and gross destruction of the $\mathrm{GH}$ joint, which most often is the preoperative state of unsuccessful arthroplasties.

In clinical practice symptomatic Larsen grade 3 shoulders with persisting synovitis should be monitored carefully and the result of conservative treatment should be evaluated regularly. When symptoms continue and radiographic findings are progressive, operative treatment should not be delayed, because treatment should not be delayed, because destruction may develop beyond the possibility of adequate repair. We emphasise that those patients with RA, who have glenohumeral erosions and a sign of upward migration of the humerus on a plain radiograph (fig 2), should be candidates for a rapid orthopaedic consultation and advanced soft tissue imaging like ultrasound or magnetic resonance arthrography.

Overall functional results of the arthroplasty are related to the physical condition as well as the motivation of the patient and the status of the rotator cuff. ${ }^{17}{ }^{24}$ Total shoulder arthroplasty will not survive without sufficient glenoid bone stock and neither will it function properly without adequately preserved soft tissue strucwithout adequately preserved soft tissue struc-
tures. ${ }^{27} \mathrm{An}$ orthopaedic consultation is worthtures. $^{27}$ An orthopaedic consultation is worth-
while at a sufficiently early stage (Larsen 3 and 4), so that timing of the operative procedure an be well planned. 1 Bjelle A. Epidemiology of shoulder problems. Baillieres Clin
Rheumatol 1989;3:437-51.
2 Lehtinen JT, Kaarela K, Belt EA, Kautiainen HJ, Kauppi Lehtinen JT, Kaarela K, Belt EA, Kautiainen HJ, Kauppi ment in seropositive rheumatoid arthritis. A 15 year endpoint study. J Rheumatol 2000;27:347-50.

3 Petersson CJ. Painful shoulders in patients with rheumatoid 5:275-9.

4 Cuomo F, Greller MJ, Zuckerman JD. The rheumatoid
shoulder. Rheum Dis Clin North Am 1998;24:67-82.

5 Green A, Norris TR. Imaging techniques for glenohumer arthritis and glenohumeral arthroplasty. Clin Orthop

6 Scutellari PN, Orzincolo C. Rheumatoid arthritis: se-
quences. Eur J Radiol 1998;27:S31-5. Crossan JF, Vallance R. The shoulder joint in rheumatoid Springer, 1982:131-9.

Dijkstra J, Dijkstra PF, van der Klundert W. Rheumatoid arthritis of the shoulder. Fortschr Röntgenstr 1985;142

9 Jónsson E. Surgery of the rheumatoid shoulder [disserta-
tion]. Lund: Lund University, 1988. Kelly IG. Unconstrained shoulder arthroplasty in rheuma-

1 Levine RB, Sullivan KL. Rheumatoid arthritis: skeletal manifestations observed on portable chest roentgeno-
grams. Skeletal Radiol 1985;13:295-303.

grams JA. The shoulder in rheumatoid arthritis. In: Lettin
Pahle J. The AWWF, Petersson C, eds. Rheumatoid arthritis surgery of the
Anher shoulder. Rheeumatology. Basel: Karger 1989,12:15-23.
Petersson C.. Shoulder surgery in rheumatoid arthritis. Acta 13 Petersson CJ. Shoulder surgery

4 Kaarela K Prognostic factors and diagnostic criteria in eaty rheumatoid arthritis. Scand J Rheumatol 1985;14(supp 57):1-54. Kaarela K, Kautiainen H. Continuous progression of radio-
logical destructine Rheumatol 1997;24:1285-7.

6 Larsen A, Dale K, Eek M. Radiographic evaluation of rheureference films. Acta Radiol Diagn 1977; 18:481-9.

7 Figgie HE, Inglis AE,Goldberg VM, Ranawat CS, Figg MP, Wile JM. An analysis of factors affecting the long-term 18 Hirooka A, Wakitani S, Yoneda M, Ochi T. Should destruction in rheumatoid arthritis. Acta Orthop Scan

Lehtinen TT, Belt EA, Lybäck CO, Kauppi MJ, Kaarela K,
Kautiainen HJ, et al Subacromial space in rheumatoid shoulder. A radiographic 15 year follow up study of 148 Soulder. A radiographic 15 year follow up study of
shoulders. Shoulder Elbow Surg 2000;9:183-7. 
20 Weiner DS, Macnab I. Superior migration of the humeral rotato cuff. J Bone Joint Surg Br 1970;52:524-7.
rotar of the Amis A. A. Biomechanics: unper limb. 4. Part 1. Upper limb
function, shoulder and elbow. Current Orthopaedics 1990; 4:21-6.
Mullaji AB, Beddow FH, Lamb GHR. CT measurement of
genoid erosion in arthritis. J Bone Joint Surg Br 1994; 76: glenoid erosion in arthritis. J Bone Joint Surg Br 1994;76:
384-8. $384-8$.
23 Rozing PM. Surgery of the rheumatoid shoulder. Rheuma23 Rozing PM. Surgery of the rheumatoid shoulder. Rheuma-

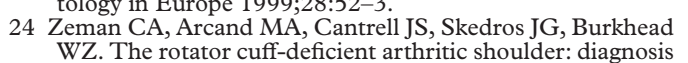

and surgical management. J Am Acad Orthop Surg 5 Resnick D, Niwayama G, eds. Diagnosis of bone and joint disorders. 2nd ed. Rheumatoid arthritis. Philadeldphia: Saunders, 1988. Kelly IG. Surgery of the rheumatoid shoulder. Ann Rheur Dising PM, Brand

Rozing PM, Brand R. Rotator cuff repair during shoulder arthroplasty in rheumatoid arthritis. $J$

Nguen VD. Rapid destructive arthritis of the shoulder. Skel-
etal Radiol 1996;25:107-12. 